\title{
High patient socioeconomic deprivation does not inhibit communication of concerns in head and neck cancer review clinics
}

\author{
Allen $S^{\text {a }}$, Harris $\mathbf{R}^{\mathrm{b}}$, Brown $S L^{\mathrm{c}}$, Humphris $\mathbf{G}^{\mathrm{d}}$, Zhou $\mathbf{Y}^{\mathrm{d}}$, Rogers $\mathbf{S N}^{\mathrm{ef}}$
}

Miss Sarah Allen, BSc (Hons.) MSc MBPsS ${ }^{\mathrm{a}}$

Department of Health Services Research, Institute of Psychology Health and Society, University of Liverpool, Room 111, $1^{\text {st }}$ floor, Block B, Waterhouse Building, 1-5 Brownlow Street, Liverpool, L69 3GL, UK

Sarah.Allen@liverpool.ac.uk

Professor Rebecca V Harris ${ }^{\mathrm{b}}$

BDS PhD Department of Health Services Research, Institute of Psychology Health and Society, Block B, Waterhouse Building, 1-5 Brownlow Street, Liverpool, L69 3GL, UK. harrisrv@liverpool.ac.uk

Dr Stephen L. Brown $\mathrm{PhD}^{\mathrm{c}}$

Department of Psychological Sciences, University of Liverpool, Liverpool, L69 3BX, UK

S.L.Brown@liverpool.ac.uk

Professor Gerry Humphris ${ }^{\mathrm{d}}$

School of Medicine, University of St Andrews, St Andrews, Fife, KY16 9TF St Andrews, Scotland, UK. gmh4@st-andrews.ac.uk

Dr Yuefang Zhou ${ }^{\mathrm{d}}$

School of Medicine, University of St Andrews, St Andrews, Fife, KY16 9TF St Andrews, Scotland, UK. yz10@st-andrews.ac.uk

Professor Simon N Rogers, FDS RCS FRCS MD

Evidence-Based Practice Research Centre (EPRC), Faculty of Health and Social Care, Edge Hill University, St Helens Road, Ormskirk, L39 4QP, ${ }^{\mathrm{f}}$ Consultant Regional Maxillofacial Unit, University Hospital Aintree, Liverpool, L9 1AE, UK. simonn.rogers@aintree.nhs.uk

Address for correspondence:

Miss Sarah Allen, Department of Health Services Research, Institute of Psychology Health and Society, University of Liverpool, Room 111, $1^{\text {st }}$ floor, Block B, Waterhouse Building, 1-5 Brownlow Street, Liverpool, L69 3GL, UK Sarah.Allen@liverpool.ac.uk Tel: 01517955317 


\section{Acknowledgments}

Sarah Allen is funded by The National Institute for Health Research Collaboration for Leadership in Applied Health Research and Care North West Coast (NIHR CLAHRC NWC) Sarah Allen is a PhD student at the NIHR CLAHRC NWC. The views expressed are those of the author(s) and not necessarily those of the NHS, the NIHR or the Department of Health. 


\title{
High patient socioeconomic deprivation does not inhibit communication of concerns in head and neck cancer review clinics
}

\begin{abstract}
Objective: To examine associations between SES and the extent to which head and neck cancer patients expressed concerns to surgeons during routine follow-up clinics.

Methods: 110 head and neck review consultations with one consultant were audiorecorded and analysed using the Verona Coding Definitions of Emotional Sequences (VRCoDES) to measure doctor-patient communication. English Indices of Multiple Deprivation (IMD) 2015 scores were grouped into deciles so that the VRCoDES could be compared by patient SES. Results: There were no significant correlations between IMD decile and the number and type of cues and concerns or the type of healthcare provider responses. There was a positive correlation between IMD decile and appointment length, $r=.288, p<.01$. When appointment length was controlled for, there was a negative correlation between IMD decile and number of cues and concerns, $r=-.221, p<.05$.
\end{abstract}

Conclusion: These findings question the assumption that SES is associated with patients' willingness to express their concerns during consultations. Shorter consultations suggest that less time is invested in either providing elaborate answers to patients' concerns or with rapport building. Strategies which facilitate rapport building with low SES patients may prove useful for clinicians to adopt.

Keywords: Doctor-patient Communication; Head and Neck Cancer; Deprivation; VRCoDES; Indices of Multiple Deprivation 


\section{Introduction}

Head and neck cancer can result in a range of long-term concerns for the patient including pain, xerostomia, fear of recurrence, disfigurement, and problems with feeding and speech (1). Research suggests that low socioeconomic status (SES) individuals are more likely to develop head and neck cancer than those higher up the socioeconomic gradient (2). Additionally, low SES patients experience poorer quality of life and survival rates $(3,4)$.

Patient-centred styles of doctor-patient communication involve clinicians interacting with patients in ways that encourage patients to take more active roles in raising and discussing issues of importance to them and participating in decision-making (5). Research suggests that patient participation is linked to positive patient outcomes such as improved quality of life, reduced anxiety and depression, greater satisfaction, better sharing of information, and better treatment adherence (6-9).

However studies have found that doctor-patient communication differs depending upon the patient's SES (10). Low SES patients tend to participate less actively in consultations, for example asking fewer questions, as well as being less likely to express emotions and spontaneously volunteer information (11). Furthermore healthcare professionals tend to involve low SES patients less in their consultations by giving them less information, spending less time building rapport with them and listening less attentively $(9,11,12)$.

Level of Deprivation (LoD) is an indicator of SES, used in the UK, based on 7 variables which together form a measure of area-level deprivation. These include housing quality, employment rates, crime and living environment present in an individual's area of residence (13). LoD is the primary indicator of area-based socioeconomic disadvantage used in the UK 
because it reflects inequalities over a broad range of social indicators. High LoD is associated with greater morbidity and mortality in individuals (14), prevalence of mental disorders (15), obesity and smoking rates (16), and poorer self-reported health (16).

Few studies have explored the effect of socioeconomic differences on patient communication with head and neck cancer patients, and none have used LoD as a measure of SES. The UK National Health Service provides theoretically equal access to health services across the SES spectrum, allowing a systematic comparison between higher and lower SES individuals. Therefore the present study aims to explore how area-level deprivation is associated with doctor-patient communication in head and neck oncology review clinics. This will contribute to our understanding of how deprivation affects doctor-patient communication in this patient group.

\section{Material and methods}

We examined associations between LoD and the incidence and timings of patients' expressions of their problems in 110 audiorecorded head and neck oncology review consultations with a single consultant Head and Neck surgeon.

Patient SES was measured using Indices of Multiple Deprivation (17) scores which were split into deciles. IMD scores are composed of aggregated summaries of income, employment, education, health, crime, access to housing and services, and living environment. IMD scores pertain to areas of an average 1,500 people in England. They are publicly available on the Department for Communities and Local Government website, and were accessed using patient postcodes (18). 
The audiotapes were analysed using the Verona Coding Definitions of Emotional Sequences (VRCoDES), which quantify patient expressions of worry or concern in a medical consultation along with the healthcare provider's responses (19-21). Patient utterances are coded as either cues or concerns: concerns are clear expressions of emotions which are explicitly verbalised, whereas cues are verbal or nonverbal hints at unpleasant emotions. There are different types of cues and each cue or concern is coded in terms of whether it was elicited by the patient or the healthcare professional (20). Healthcare provider responses are coded in terms of explicitness and providing or reducing space for further emotional disclosure; in other words, whether the healthcare provider encourages or discourages the patient from talking further about their emotional concerns, and whether they do so in a direct or indirect manner (19). VRCoDES have been used to study doctor-patient communication in a variety of healthcare settings and with numerous different patient groups $(22,23)$.

Pearson's correlation was used to examine associations between IMD decile and number of cues and concerns, elicitation, and type of consultant response. Pearson's partial correlation was used to control for appointment length.

\section{Results}

The mean age of the sample was 62.9 years $(\mathrm{SD}=12.69)$, with a range of $29-93$ years, and most of the sample was male (63.6\%). The mean appointment length was 9 minutes 17 seconds, with a range of 3 minutes 52 seconds to 21 minutes 55 seconds. Tables 1 and 2 outline the clinical characteristics of the sample and the distribution of IMD deciles.

Mean number of cues and concerns can be found in Table 3. As can be seen in Table 4 there were no significant correlations between IMD decile and number of cues and concerns, 
elicitation of cues and concerns, clinician responses to cues and concerns, type of cues and proportion of concerns to cues.

There was however a significant positive correlation between IMD decile and length of appointment, $r=.288, p<.01$. In addition, when length of appointment was controlled using a Pearson's partial correlation, a significant and negative association between number of cues and concerns and IMD decile was found, $r=-.221, p<.05$. There was a positive correlation between IMD decile and time to first cue or concern which approached significance, $r=.218$, $p=.077$.

\section{Discussion}

In the present study no significant correlations were found between IMD decile and number of cues and concerns, elicitation, or consultant responses. This is in contrast to findings from previous studies such as Siminoff et al.(11) which found that higher education and income patients asked more questions, received more information and experienced greater rapport with clinicians. Our study used LoD as an area-based indicator of SES rather than education and income which are individual-level variables. This suggests that perhaps the influence of SES on doctor-patient communication in this particular patient group is limited. Previously reported SES effects of education and income on communication may pertain to the specific influence of verbal or other academic skills rather than SES, per se.

When consultation length was controlled however, a significant negative association was found between IMD decile and number of cues and concerns. This does not show that lower SES patients expressed more cues and concerns, but that a greater amount of time was taken in discussions between patients and their consultant during which patients did not express 
cues or concerns. There may have been greater general discussion and rapport building. This interpretation is supported by the non-significant effect whereby lower SES patients took a shorter time to broach their first cue of concern. It may be that low SES patients are quick to express any issues to their consultant, with relatively little small-talk around these or other issues.

Such an interpretation fits with previous research finding that healthcare professionals spend more time building rapport with high SES patients (11), and adds the insight that it may be the patient not the doctor who curtails rapport building by moving towards problem solving. Nonetheless, building rapport is an important aspect of clinical communication with a number of benefits for patients $(5,7-9)$, and low SES patients may be placed at a disadvantage if less time is spent building rapport.

Alternatively, higher SES patients may have received more elaborate clinician responses to their cues and concerns, although we note that the clinician did not favour higher or lower SES groups in eliciting concerns. This is in line with a previous study on patient preferences for communication style (24). The Aelbrecht et al. (24) study found that low educated patients attached most importance to affective elements of a consultation, whereas middle and high educated patients felt that problem-solving was more important. It may be that in the present study high SES patients were more interested in receiving information and engaging in problem-solving during the consultation.

There are a number of limitations to the present study which may have affected the findings. Firstly only 110 audiotapes were analysed as part of this study, therefore the sample size may have been too small to detect any significant differences. Future studies should try to 
replicate this with larger samples in order to determine if socioeconomic differences in doctor-patient communication remain absent. Secondly, only one consultant participated in the present study, compromising our ability to generalise these findings. On the other hand the use of only one consultant in this study allowed the authors to control for variation between consultants, therefore isolating the influence of patient's deprivation on doctorpatient communication. There are some limitations to using IMD as a measure of SES. As IMD scores pertain to postcodes, it is possible that although an individual may live in an area with a high level of deprivation they may be high SES in terms of education or occupation.

\section{Conclusion}

The absence of significant correlations between IMD decile and number of cues and concerns, as well as consultant responses suggests that perhaps SES, assessed by geographic location, is a weaker indicator associated with clinical communication quality than individual-level variables such as education (11). However, our findings suggest that less communication exists between lower SES patients and their clinician, possibly because patients are quicker to broach their concerns. Whilst we acknowledge that more communication is not necessarily better communication (25), it may be advantageous for clinicians to adopt active strategies that allow them to build rapport with lower SES patients who are keen to voice their concerns.

\section{Conflict of Interest}

None 


\section{Ethics statement/confirmation of patient permission}

All procedures performed in this study were approved by and in accordance with the ethical standards of the Clinical Audit Department, University Hospital

, University of

School of Medicine and with the 1964 Helsinki declaration and its later

amendments or comparable ethical standards. This article does not contain any studies with animals performed by any of the authors. Informed consent was obtained from all individual participants included in the study.

\section{References}

1. Rogers SN. Quality of life perspectives in patients with oral cancer. Oral Oncology. 2010;46(6):445-7.

2. Auluck A, Walker BB, Hislop G, Lear SA, Schuurman N, Rosin M. Population-based incidence trends of oropharyngeal and oral cavity cancers by sex among the poorest and underprivileged populations. BMC Cancer. 2014;14:11.

3. Allen S, Lowe D, Harris RV, Brown S, Rogers SN. Is social inequality related to different patient concerns in routine oral cancer follow-up clinics? Eur Arch Oto-RhinoLaryn. 2016:1-9.

4. Rylands J, Lowe D, Rogers SN. Outcomes by area of residence deprivation in a cohort of oral cancer patients: Survival, health-related quality of life, and place of death. Oral oncology. 2016;52:30-6.

5. Epstein RM, Fiscella K, Lesser CS, Stange KC. Why The Nation Needs A Policy Push On Patient-Centered Health Care. Health Aff. 2010;29(8):1489-95.

6. Morris J, Royle GT. OFFERING PATIENTS A CHOICE OF SURGERY FOR EARLY BREAST-CANCER - A REDUCTION IN ANXIETY AND DEPRESSION IN PATIENTS AND THEIR HUSBANDS. Soc Sci Med. 1988;26(6):583-5. 
7. Arora NK. Interacting with cancer patients: the significance of physicians' communication behavior. Soc Sci Med. 2003;57(5):791-806.

8. Street RL, Voigt B. Patient participation in deciding breast cancer treatment and subsequent quality of life. Medical Decision Making. 1997;17(3):298-306.

9. Hall JA, Roter DL, Katz NR. META-ANALYSIS OF CORRELATES OF PROVIDER BEHAVIOR IN MEDICAL ENCOUNTERS. Medical Care. 1988;26(7):657-75. 10. Verlinde E, De Laender N, De Maesschalck S, Deveugele M, Willems S. The social gradient in doctor-patient communication. Int J Equity Health. 2012;11(1).

11. Siminoff LA, Graham GC, Gordon NH. Cancer communication patterns and the influence of patient characteristics: disparities in information-giving and affective behaviors. Patient Educ Couns. 2006;62(3):355-60.

12. Martin E, Russell D, Goodwin S, Chapman R, North M, Sheridan P. WHY PATIENTS CONSULT AND WHAT HAPPENS WHEN THEY DO. Br Med J. 1991;303(6797):289-92.

13. Macintyre S, Maciver S, Sooman A. Area, class and health: should we be focusing on places or people? Journal of social policy. 1993;22(02):213-34.

14. Reijneveld SA, Verheij RA, de Bakker DH. Relative importance of urbanicity, ethnicity and socioeconomic factors regarding area mortality differences. J Epidemiol Community Health. 1999;53(7):444-5.

15. Reijneveld SA, Schene AH. Higher prevalence of mental disorders in socioeconomically deprived urban areas in the Netherlands: Community or personal disadvantage? J Epidemiol Community Health. 1998;52(1):2-7.

16. Reijneveld SA. The impact of individual and area characteristics on urban socioeconomic differences in health and smoking. Int J Epidemiol. 1998;27(1):33-40. 17. The English Indices of Deprivation 2015. In: Government DfCaL, editor. 2015. 
18. The English Indices of Deprivation 2010. In: Government DfCaL, editor. 2011.

19. Del Piccolo L, de Haes H, Heaven C, Jansen J, Verheul W, Bensing J, et al.

Development of the Verona coding definitions of emotional sequences to code health providers' responses (VR-CoDES-P) to patient cues and concerns. Patient Educ Couns. 2011;82(2):149-55.

20. Zimmermann C, Del Piccolo L, Bensing J, Bergvik S, De Haes H, Eide H, et al. Coding patient emotional cues and concerns in medical consultations: The Verona coding definitions of emotional sequences (VR-CoDES). Patient Educ Couns. 2011;82(2):141-8.

21. Piccolo LD, Finset A, Mellblom AV, Figueiredo-Braga M, Korsvold L, Zhou Y, et al. Verona Coding Definitions of Emotional Sequences (VR-CoDES): Conceptual Framework and Future Directions. Patient Educ Couns.

22. Del Piccolo L, Pietrolongo E, Radice D, Tortorella C, Confalonieri P, Pugliatti M, et al. Patient Expression of Emotions and Neurologist Responses in First Multiple Sclerosis Consultations. PLoS One. 2015;10(6):12.

23. Finset A, Heyn L, Ruland C. Patterns in clinicians' responses to patient emotion in cancer care. Patient Educ Couns. 2013;93(1):80-5.

24. Aelbrecht K, Rimondini M, Bensing J, Moretti F, Willems S, Mazzi M, et al. Quality of doctor-patient communication through the eyes of the patient: variation according to the patient's educational level. Advances in Health Sciences Education. 2015;20(4):873-84.

25. Elmore N, Burt J, Abel G, Maratos FA, Montague J, Campbell J, et al. Investigating the relationship between consultation length and patient experience: a cross-sectional study in primary care. British Journal of General Practice. 2016;66(653):E896-E903. 


\begin{tabular}{|l|l|}
\hline IMD decile & Percent of sample \\
\hline 1 & 30.9 \\
\hline 2 & 9.1 \\
\hline 3 & 4.5 \\
\hline 4 & 1.8 \\
\hline 5 & 10 \\
\hline 6 & 9.1 \\
\hline 7 & 10 \\
\hline 8 & 10 \\
\hline 9 & 8.2 \\
\hline 10 & 6.4 \\
\hline
\end{tabular}

Table 1. Distribution of IMD decile in sample

\begin{tabular}{|c|c|c|c|}
\hline \multicolumn{2}{|l|}{ Treatment } & \multicolumn{2}{|c|}{ Percent of sample } \\
\hline \multicolumn{2}{|l|}{ Surgery only } & \multicolumn{2}{|c|}{40.9} \\
\hline \multicolumn{2}{|c|}{ Surgery with radiotherapy } & \multicolumn{2}{|l|}{52.7} \\
\hline \multicolumn{2}{|c|}{ Radio/chemoradiotherapy without surgery } & \multicolumn{2}{|c|}{6.4} \\
\hline \multicolumn{2}{|c|}{ Stage at diagnosis } & \multicolumn{2}{|c|}{ Percent of sample } \\
\hline \multicolumn{2}{|l|}{ Early } & \multicolumn{2}{|c|}{50} \\
\hline \multicolumn{2}{|l|}{ Late } & \multicolumn{2}{|l|}{31.8} \\
\hline \multicolumn{2}{|l|}{ Missing } & \multicolumn{2}{|c|}{18.2} \\
\hline \multicolumn{2}{|l|}{ Primary cancer site } & \multicolumn{2}{|c|}{ Percent of sample } \\
\hline \multicolumn{2}{|l|}{ Oral } & \multicolumn{2}{|c|}{51.8} \\
\hline \multicolumn{2}{|l|}{ Pharyngeal } & \multicolumn{2}{|l|}{25.5} \\
\hline \multicolumn{2}{|l|}{ Other } & \multicolumn{2}{|l|}{22.7} \\
\hline & Mean & $\begin{array}{l}\text { Standard } \\
\text { deviation }\end{array}$ & Range \\
\hline $\begin{array}{l}\text { Time since diagnosis } \\
\text { (months) }\end{array}$ & 56.85 & 51.74 & $6-240$ \\
\hline
\end{tabular}

Table 2. Clinical characteristics of sample

\begin{tabular}{|l|l|l|l|l|l|}
\hline & Mean & $\underline{\text { Median }}$ & Standard deviation & Range & $\underline{\text { QQR }}$ \\
\hline $\begin{array}{l}\text { Total number of } \\
\text { cues and concerns }\end{array}$ & 1.81 & $\underline{1.00}$ & 2.4 & $0-13$ & $\underline{3}$ \\
\hline $\begin{array}{l}\text { Total number of } \\
\text { cues }\end{array}$ & 1.08 & $\underline{0}$ & 1.69 & $0-8$ & $\underline{2}$ \\
\hline Number of concerns & 0.73 & $\underline{0}$ & 1.2 & $0-8$ & $\underline{1}$ \\
\hline Number of cue a & 0.23 & $\underline{0}$ & 0.55 & $0-3$ & $\underline{0}$ \\
\hline Number of cue b & 0.65 & $\underline{0}$ & 1.08 & $0-5$ & $\underline{1}$ \\
\hline Number of cue c & 0.03 & $\underline{0}$ & 0.16 & $0-1$ & $\underline{0}$ \\
\hline Number of cue d & 0.15 & $\underline{0}$ & 0.41 & $0-2$ & $\underline{0}$ \\
\hline Number of cue g & 0.02 & $\underline{0}$ & 0.19 & $0-2$ & $\underline{0}$ \\
\hline
\end{tabular}

Table 3. Mean number of cues and concerns per consultation

No instance of cue e and $f$ were observed 


\begin{tabular}{|l|l|l|}
\hline & $R$ & $P$ \\
\hline Number of cues and concerns & -.004 & .97 \\
\hline $\begin{array}{l}\text { Number of patient elicited } \\
\text { cues and concerns }\end{array}$ & .072 & .456 \\
\hline $\begin{array}{l}\text { Number of consultant elicited } \\
\text { cues and concerns }\end{array}$ & -.059 & .543 \\
\hline Time to first cue or concern & .218 & .077 \\
\hline $\begin{array}{l}\text { Number of explicit reduce } \\
\text { space responses }\end{array}$ & .03 & .756 \\
\hline $\begin{array}{l}\text { Number of explicit provide } \\
\text { space responses }\end{array}$ & -.083 & .389 \\
\hline $\begin{array}{l}\text { Number of non-explicit reduce } \\
\text { space responses }\end{array}$ & .004 & .97 \\
\hline $\begin{array}{l}\text { Number of non-explicit } \\
\text { provide space responses }\end{array}$ & .055 & .566 \\
\hline Appointment length & .288 & $<.01$ \\
\hline Number of cue a & -.091 & .346 \\
\hline Number of cue b & .02 & .836 \\
\hline Number of cue c & -.05 & .603 \\
\hline Number of cue d & -.074 & .445 \\
\hline Number of cue g & .072 & .456 \\
\hline Proportion of concerns to cues & .009 & .943 \\
\hline
\end{tabular}

Table 4. Correlation coefficients and $p$ values for IMD deciles 